\title{
Small Area Analysis of Hospital Discharges for Musculoskeletal Diseases in Michigan: The Influence of Socioeconomic Factors
}

\author{
LAURENCEF. MCMahoN, Jr., M.D., M.P.H., CATHERINe G. MCLAughlin, Ph.D., \\ Gina R. Petroni, Ph.D., PHILIP J. TedesChI, Ph.D., Ann Arbor, Michigan
}

PURPOSE: The rise in health care costs has occasioned a number of initiatives in an attempt to reduce the rate of increase. Despite the growth of health maintenance organizations and preferred provider organizations and the introduction of Medicare's prospective payment system, health care costs have continued to increase. Coincident with these efforts, a number of researchers have shown that there exists wide variation in age-adjusted hospital discharge rates, which translate into significant variation in per capita expenditures. Much of the focus on the reasons for hospital admission variability has been on physician practice variation. If most of the variation in hospital discharge rates is due to physician practice style, then payment systems can be developed (e.g., capitation) that limit physician practice variation without harming patients. We examined socioeconomic factors in Michigan communities to assess their association with hospital discharge rates for patients with musculoskeletal diseases.

PATENTS AND METHODS: Data on hospital discharges from 1980 and 1987 were taken from the Michigan Inpatient Data Base. All admissions from the major diagnostic category 8, diagnosisrelated group (DRG) 209-256 were included. Zip code-specific hospitalization data were grouped into small geographic areas or hospital market communities (HMCs). Discharge rates were calculated, and profiles of the socioeconomic characteristics of each of the HMCs were developed. A Poisson regression model with an extrasystematic component of variance was used to ana-

From the Departments of Internal Medicine (LFM), School of Medicine Health Services Management and Policy (LFM, CGM, PJT), and Biostatistics (GRP), School of Public Health, University of Michigan, Ann Arbor, Michigan.

This work was presented in part at the American Federation for Clinical Research Meeting, Washington, D.C., May 1989.

Financial support was provided by UM-MAC Grant 5 P60 AR20557 from the National Institutes of Health.

Requests for reprints should be addressed to Laurence F. McMahon, Jr., M.D., M.P.H., Division of General Medicine, Department of Internal Medicine, 3116 Taubman Center, 1500 East Medical Center Drive, University of Michigan, Ann Arbor, Michigan 48109-0376.

Manuscript submitted August 23,1990, and accepted in revised form April 11, 1991. lyze the association of HMC socioeconomic characteristics with age-adjusted hospital use.

RESULTS: We found that four socioeconomic variables, average annual income per capita, percent of the population with four years of college, percent of the population living in an urban area, and percent of families with incomes below the poverty line, explained $26.6 \%\left(\mathbf{R}^{2}\right)$ of the variation in overall hospital discharge rates (p < 0.001). Moreover, we found that the ability of the model to explain variability was influenced by the type of disease, and that these socioeconomic variables had a consistent effect across the range of DRGs. Finally, we noted that, over the period of 1980 to 1987 , socioeconomic factors remained important in explaining hospital use despite the dramatic changes in the delivery of care over this period.

CONCLUSION: Socioeconomic factors play a significant role in explaining the observed variation in hospital discharge rates for musculoskeletal diseases. Models utilixing only physician practice variation to account for the populationbased differences in discharge rates are overly simplistic. In order to ensure that vulnerable subsets of the population are not harmed by the introduction of cost-containment strategies based on simplistic models, more attention must be paid to the socioeconomic and epidemiologic factors related to hospital use.

$\mathbf{O}$ ver the period from 1975 to 1987 , health care costs have risen from 133 billion dollars or $8.3 \%$ of our gross national product to 500 billion dollars or $11.1 \%$ of our gross national product [1]. Treatment of patients with musculoskeletal problems accounts for a significant proportion of our nation's health care cost. As noted in the National Medical Care Utilization and Expenditure Survey (NMCUES), patients with these conditions represent approximately $20 \%$ of the non-institutionalized population. Total charges for the treatment of these conditions accounted for more than 12 billion dollars or $8 \%$ of the nation's total health care costs 
of the civilian non-institutionalized population in 1980 . While approximately $40 \%$ of all health care expenditures are related to hospital care, according to the NMCUES, $59 \%$ of charges for musculoskeletal conditions in 1980 occurred in an in-hospital setting, demonstrating the importance of hospitalbased care for patients with musculoskeletal diseases [2].

A number of investigators have identified the fact that there exists significant variation in the ageadjusted hospitalization rates for various medical conditions and surgical procedures between geographic areas, mainly hospital service areas and counties [3-5]. These significant differences in the rates of admission can vary by factors ranging from 2 to 8 between the highest and lowest, and account for significant differences in the per capita expenditures between small areas [6]. Given the interest in cost control, health policy makers are eager to reduce the rates in high-use areas to those of the lowuse areas. A key clinical question stemming from such variation in the use of hospitals is, "Are these differences in rates clinically understandable or are they due mainly to differences in physician practice variation?" If differences in hospital admission rates are due principally to physician practice variation, then instituting capitation models for payment may lead to the desired reduction in hospital admission rates in high-use areas with no deleterious health effects. If, however, hospitalization rates are related to patient or community-specific characteristics (e.g., severity of illness, poverty, access to facilities, and the like), then capitation systems that do not control for such factors may place the population at risk of underservice [7-9].

We examined the variation in small area hospital admissions in 1980 and again in 1987 for musculoskeletal diseases in the state of Michigan and explored the relationship between hospital discharge rates and the socioeconomic characteristics of the communities.

\section{PATIENTS AND METHODS}

\section{Patient Population}

Hospital discharges identified for this study were taken from the Michigan Inpatient Data Base (MIDB). The MIDB is a computerized file of all Michigan residents who were hospitalized in Michigan and border communities in Ohio and Indiana. Data from 1980 and 1987 were utilized in the current study. The 1980 hospitalization data were selected to enable the use of socioeconomic data collected as part of the 1980 census. In 1980 , there were 1.5 million hospitalizations in the more than 200 Michigan and border community hospitals. All ad- missions from the major diagnostic category (MDC) 8, Diseases and Disorders of the Musculoskeletal System and Connective Tissue (diagnosis-related group [DRG] 209-256), were included-a total of 140,573 admissions in 1980 , and 92,685 admissions in 1987.

\section{Definition of Small Geographic Areas}

Zip code-specific hospitalization data were grouped into small geographic areas or Hospital Market Communities (HMCs) according to where the plurality of residents in the zip code area sought hospital care. The definition of an HMC was a twostep process. First, hospitals in the same or nearby towns were grouped together using a computer algorithm designed to identify hospitals with significant market overlap. These groups of hospitals were then reviewed for reasonableness by panels of administrators and health planners familiar with the area. Second, each zip code area was assigned to the hospital, or group of hospitals, having the largest single fraction (plurality) of its total use. Zip code areas that form the HMCs consist of contiguous zip code areas [10].

Application of these techniques led to the formation of $60 \mathrm{HMCs}$ in the lower peninsula of Michigan, a population of 8.9 million in 1980 . The HMCs range in population from 9,108 to 839,410 , and include from one to 16 hospitals. The average HMC has a population of 147,650 and 3.75 hospitals. The composition of HMCs appears stable over time. Eighty-seven percent of zip code areas held the same plurality assignment from 1980 to 1985 . Only eight of the $60 \mathrm{HMCs}$ had mean absolute changes in market share exceeding $3 \%$ per year.

\section{Discharge Rate Calculation}

Age-adjusted HMC-based discharge rates were determined for the $48 \mathrm{DRGs}$ in MDC 8. For each DRG, age-specific discharge rates were calculated for the state. These DRG statewide discharge rates were applied to the age-specific population in each HMC to yield an expected number of discharges. The ratio of the observed number of discharges to the expected number yields a standardized discharge ratio. The product of the statewide crude discharge rate of the DRG and the DRG-specific standardized discharge ratio yields the indirect standardized discharge rate for that HMC. Hospital discharges were included in the HMC-based calculations wherever they actually occurred. Thus, the HMC-based discharge calculation includes both cases admitted to hospitals in the HMC and patients from the HMC admitted to hospitals in any other HMC. 


\section{HMC Socioeconomic Characteristics}

We developed profiles of the socioeconomic characteristics of each of the $60 \mathrm{HMCs}$ by aggregating zip code-specific socioeconomic data obtained from the Bureau of the Census, Standard Tape File (STF) 3-B, 1981. These zip code-specific data were then aggregated to the HMC to develop the profile of socioeconomic characteristics of the HMCs.

The variation in discharge rates among HMCs consists of two elements, random variation and actual differences in discharge rates between communities. It is very important to distinguish between random variability and variability related to differences in population characteristics. The Poisson regression with an extra systematic component of variance enables us to do this [11,12]. Models are reported as significant if $p<0.05$.

A Poisson regression model with an extra systematic component of variance was used to analyze these data as follows. Let $i$ index HMC, and $Y_{i}$ denote the number of discharges, $E_{i}$ denote the indirect age-adjusted expected number of discharges [13], and $z_{i}$ denote the vector of socioeconomic characteristics, for the $i^{t h}$ HMC. The number of discharges $Y_{i}$ is assumed to have a Poisson distribution with mean $\mu_{i}$. The log-linear model, $\log \left(\mu_{i}\right)=\log$ $\left(E_{i}\right)+\beta^{\prime} z_{i}+f_{i}$, accounts for both the systematic and extra-Poisson variability, where $\beta^{\prime} z_{i}$ denotes a linear combination of the socioeconomic characteristics of the HMC, and $f_{i}$ denotes a random component that accounts for the fact that the mean in an HMC cannot be completely specified in terms of the socioeconomic characteristics. For interpretation of the results in terms of discharge rates, the model can be rewritten as $\mu i / E_{i}=e \beta^{\prime} z_{i}+f_{i}$.

\section{RESULTS}

\section{Descriptive}

The overall HMC age-adjusted hospital discharge rate for musculoskeletal disease in Michigan in 1980 was 172.0 per 10,000 population with a standard deviation of 34.5. The discharge rates ranged from a high of 256.5 to a low of 107.0 per 10,000 , yielding a ratio of 2.40 highest to lowest. The ratio of the highest to the lowest rate is consistent with that seen in other diseases [6].

The overall HMC age-adjusted hospital discharge rate for musculoskeletal disease in 1987 was 113.5 per 10,000 population with a standard deviation of 15.8. The 1987 discharge rates ranged from a high of 162 to a low of 76 per 10,000 , yielding a ratio of 2.13 from highest to lowest.

The overall mean 1980 discharge rate for medical musculoskeletal cases (DRGs 235-256) was 84.5 with a standard deviation of 24.8 per 10,000 population. The medical discharges ranged from 49.9 to 145.1 per 10,000 population with a ratio of 2.90 from the highest to the lowest rates. The overall mean 1980 surgical discharge rate (DRGs 209-234) was 87.4 with a standard deviation of 17.1 per 10,000 population, and ranged from 41.8 to 128.5 with a highest to lowest ratio of 3.07 .

The overall mean 1987 discharge rate for medical musculoskeletal cases (DRGs 235-256) was 46.8 with a standard deviation of 11.6 per 10,000 population. The medical discharges ranged from 18.8 to 80.2 per 10,000 population with a ratio of 4.26 from highest to lowest rates. The overall mean 1987 surgical discharge rate (DRG 209-234, 471) was 66.7 with a standard deviation of 11.2 per 10,000 population, and ranged from 47.6 to 95.5 with a highest to lowest ratio of 2.00 .

\section{Overall Regression Analysis}

We analyzed the effect of four socioeconomic variables on the variation in HMC discharge rates: average annual income per capita, the percent of the population 25 years or older with four years of college, the percent of the population living in an urban area, and the percent of families with annual income below the poverty line. Utilizing the Poisson regression model with the extra systematic component of variation, we analyzed the effect of these four variables on age-adjusted hospital discharges.

The four independent variables explained $26.6 \%$ $\left(R^{2}\right)$ of the variation in overall discharge rates for musculoskeletal disease in Michigan in 1980 (p $<0.001$ ). These results varied, however, for medical and surgical discharge rates. The model did not explain a significant amount of the variation in surgical rates $\left(\mathrm{R}^{2}=3.9 ; \mathrm{p}>0.05\right)$, but did explain a statistically significant $39.6 \%\left(\mathrm{R}^{2}\right)$ of the variation in medical admissions ( $\mathrm{p}<0.001$ ).

Utilizing the $1980 \mathrm{HMC}$ socioeconomic values and the 1987 hospital discharge data, we conducted similar Poisson regressions. The purpose of this analysis was to assess whether the dramatic changes in clinical practice that have occurred over this 7 . year period have altered the association between socioeconomic factors and hospital use identified in 1980. The same four independent variables explained $31.9 \%\left(R^{2}\right)$ of the variation in overall discharge rates for musculoskeletal disease in Michigan in 1987 ( $p<0.001$ ). In contrast to 1980 , the 1987 model explained a significant amount of the variation in surgical discharge rates $\left(R^{2}=19.5 ; p<0.05\right)$, and the model continued to explain a statistically significant percent of the variation in 1987 medical admissions $\left(\mathrm{R}^{2}=25.6 ; \mathrm{p}<0.001\right)$. 


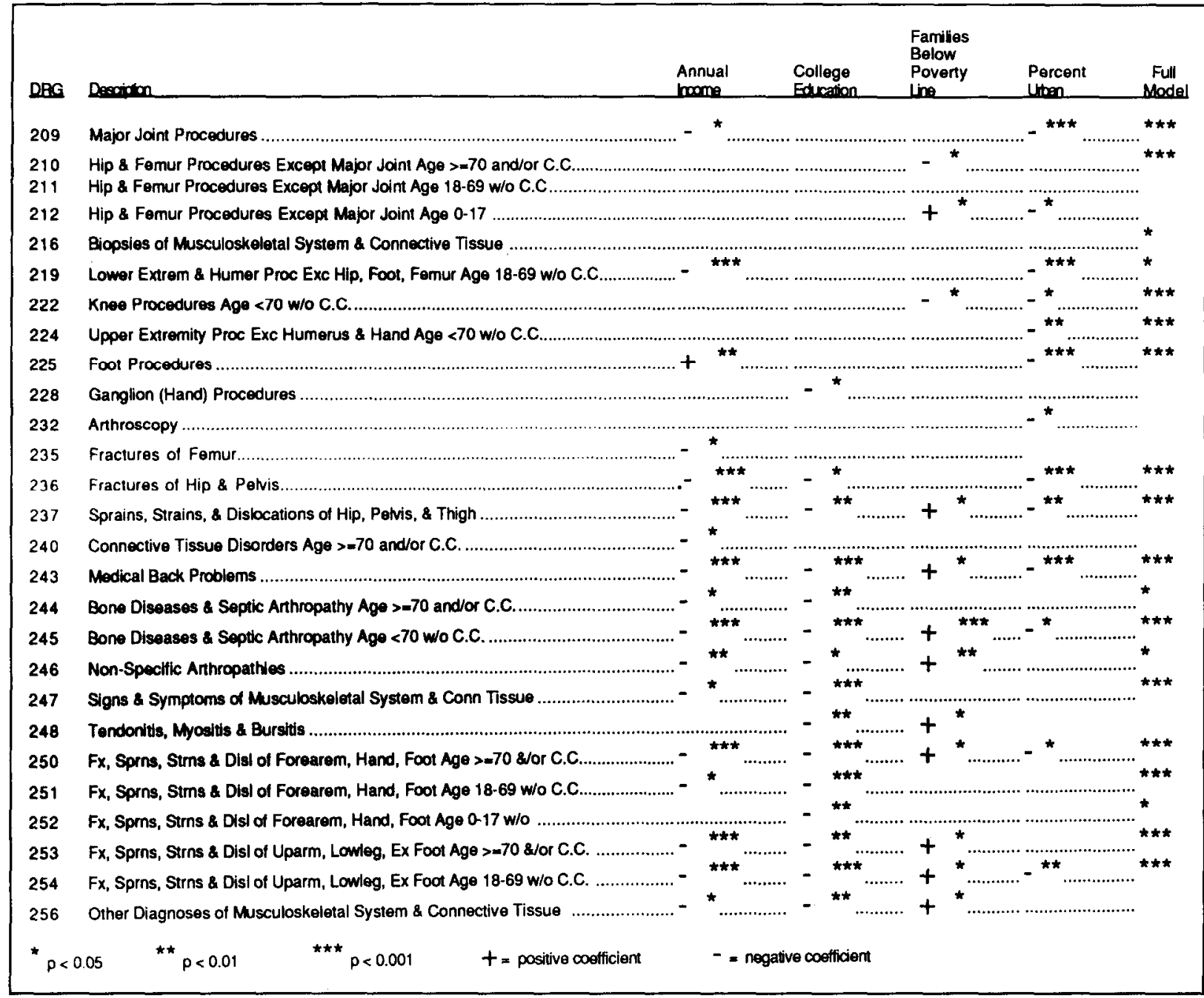

Figure 1. Results of Poisson regressions with extrasystematic component of variation in musculoskeletal DRGs (209-256) for individual independent variable models and full model.

\section{DRG-Specific Regression Analysis}

Given the fact that conditions included in the musculoskeletal DRGs represent both a wide array of severity and differing profiles of patients affected by the diseases, we hypothesized that there may be differences in the effect of socioeconomic factors by DRG. Therefore, we explored the individual effects of each of the four socioeconomic variables for each DRG. In addition, we examined the full model, that is, including all four variables, at the DRG level. Figure 1 shows the results of the DRG-specific analysis for any DRG in which any model was statistically significant in 1980 .

Annual income per capita was a significant determinant of 1980 hospital use in 17 of the 47 musculoskeletal disease DRGs. In 16 of the 17 regressions in which it was significant, the regression coefficient was negative, indicating that HMCs with aboveaverage income per capita exhibited below-average age-adjusted DRG-specific discharge rates. The one exception to this general trend was DRG 225, Foot Procedures.

The educational level of the population was a significant variable in 15 of the 47 musculoskeletal DRG models. In every case, HMCs with a higher percent of the population with a college education had lower hospital discharge rates.

The number of families below the poverty line was significant in 12 of the $47 \mathrm{DRGs}$. In general, HMCs with poorer families had higher hospital discharge rates. The exceptions to this general trend included DRG 212, Hip and Femur Procedures Except Major Joint Age $\geq 70$ and/or CC, and DRG 222, Knee Procedures.

Finally, the percent urban status of the HMC was found to be significant in 10 of the 47 DRGs, and as urbanism increased, hospital discharge rates decreased.

The final column in Figure 1 identifies those DRGs in which the full model was statistically sig- 
nificant. In 18 of $47 \mathrm{DRGs}$, the full model was found to be significant. Because of likely multicolinearity problems, we do not report the significance levels of each of the independent variables in these multiple regression models. In particular, the income level of the community is highly correlated with the other three measures of socioeconomic status.

\section{COMMENTS}

Analysis of the underlying causes of small area variation in hospital use rates is important for several reasons. First, understanding small area variation in the use of the hospital helps to elucidate the interaction between patients and the medical care system, that is, we can begin to identify the mix of motivators that come to determine whether a patient is admitted to the hospital (e.g., epidemiology of disease, socioeconomics, physician uncertainty, and the like). In addition, such analysis helps to identify potential methods to change inappropriate use rates whether too high or too low. Third, understanding the reasons that rates vary may allow us to develop new hospital payment systems (e.g., capitation), while ensuring access to those who need to be shielded from economic rationing (e.g., the poor in failing health).

Much of the small area variation analysis discussion to date has centered on physician practice variation as a principal reason for variation in the use of the hospital and has largely discounted the influence of socioeconomic factors [7] or identified suggestive associations [8]. This scenario is of concern. If all small area variation in the use of hospitals is secondary to physician-based practice variation and patient or community characteristics have no effect, then payment systems could be constructed to pay at the lowest or average rate for hospital care across all areas with no harm to patients. On the other hand, if socioeconomic factors are important in determining use, then patients could be placed at risk if payment systems failed to adjust explicitly for such factors. Moreover, a number of studies have shown that persons in different socioeconomic strata are differentially affected by certain diseases [14-16]. In addition, others have shown that the poor have problems with access to care [17]. Given this other work, our findings are in keeping with these epidemiologic and survey results.

McMahon et al [18] have demonstrated previously that the extent of poverty is an important independent variable in explaining small area variation in hospital discharge rates for gastrointestinal diseases. In this present study, we have demonstrated that four socioeconomic variables, including the ex- tent of poverty, have an effect in explaining variation in hospital discharges for musculoskeletal diseases.

Several caveats must be noted in the interpretation of our results. First, we have highlighted any model that was significant at the $p<0.05$ level. Clearly, multiple models will identify some significant associations by chance alone. We chose to use this less stringent significance level to examine the sign of the association across DRGs. Thus, high levels of annual income, education, and urbanism are associated with decreased hospitalizations, while higher concentrations of poverty are associated with more use. The exceptions to these overall trends must be viewed in a clinical context for plausibility. Finally, these DRG-specific results need to be corroborated using other databases or years of data.

Second, we have a limited number of socioeconomic variables available for analysis and no provider or delivery variables (e.g., the number of physicians or hospital beds per capita) available for inclusion in the models. These omitted socioeconomic or supply-side variables may represent the "true" determinants that account for the observed variation in discharge rates, with the included variables acting as proxy variables that are correlated with those omitted. Although supply-side variables would be important to include in the analysis, there is no accepted method that one can use to apportion such variables to HMCs that is not circular. The most frequently used method is on the basis of hospital discharges. Thus, if $X$ percent of patients in an HMC are treated in the HMC, $X$ percent of that HMC's physicians are "assigned" to the HMC to develop a physician-to-population ratio. Given how the measure was constructed, it is not surprising that this type of apportionment will identify physician supply as being important in explaining use rates [9].

Our results demonstrate that socioeconomic variables explain a significant proportion of the variance in age-adjusted population-based hospital discharges for musculoskeletal diseases. Further, we have demonstrated that the influence of specific socioeconomic variables is consistent across a wide range of DRGs, that there is a DRG-specific effect, and that the effect persists over time. Where we have identified occasional exceptions to the overall trends in the association between individual socioeconomic variables and hospital discharge rates, these results must be viewed as tentative given the multiple models that were examined.

These results identify both the importance of socioeconomic variables in understanding the differ- 
ences between hospital discharge rates among small areas, and the necessity to include such variables in capitation models or other initiatives designed to lower health care costs. For example, higher concentrations of poverty were associated with higher use rates for a number of DRGs. If these differences are not included in cost-containment efforts, a particularly vulnerable subset of patients, the poor, may suffer $[19,20]$.

\section{ACKNOWLEDGMENT}

We wish to thank Lisa Lavey for her assistance in manuscript preparation, and two anonymous reviewers for their helpful suggestions.

\section{REFERENCES}

1. Letsch SW, Levit KR, Waldo DR. National health expenditures. Health Care Financing Review 1988; 10: 109-29.

2. Murt HA, Parsons PE, Harlan WR, et al. Disability, utilization, and costs associated with musculoskeletal conditions, United States, 1980. National Medical Care Utilization and Expenditure Survey. Series C, Analytical Report No. 5. DHHS Publication No. 86-20405. National Center for Health Statistics, Public Health Service. Washington: U.S. Government Printing Office. September 1986. 3. Lewis CE. Variations in the incidence of surgery. N Engl J Med 1969; 281: 880-4.

4. Wennberg J, Gittelson A. Variation in medical care among small areas. Sci Am 1982; 246: 120-34

5. Chassin MR, Kosecoff J, Park RE, et al. Does inappropriate use explain geographic variations in the use of health care services? A study of three proce dures. JAMA 1987; 258: 2533-7.

6. Paul-Shaheen P, Clark JD, Williams D. Small area analysis: a review and analysis of the North American literature. J Health Polit Policy Law 1987; 12 $741-809$.

7. Wennberg JE, Freeman JL, Culp WJ. Are hospital services rationed in New Haven or over-utilized in Boston? Lancet 1987; 1: 1185-8.

8. Perrin JM, Homer CJ, Berwick DM, Woolf AD, Freeman JL, Wennberg JE. Variations in rates of hospitalization of children in three urban communities. $N$ Engl $J$ Med 1989; 320: 1183-7.

9. McLaughlin CG, Normolle DP. Wolfe RA, McMahon LF, Griffith JR. Small area variation in hospital discharge rates: do socioeconomic variables matter? Med Care 1989; 27: 507-21

10. Thomas JW, Griffith JR, Durance P. Defining hospital clusters and associated service communities in metropolitan areas. Socio-Economic Plan Sci 1981; 15 45-51.

11. Breslow NE. Extra-Poisson variation in log-linear models. Applied Statistics 1984; 33: 38-44.

12. Wolfe RA, Petroni GR, MCLaughlin CM, MCMahon LF. Empirical evaluation of statistical models for rates and counts. Stat Med (in press)

13. Breslow NE, Lubin JH, Marek P, Langholtz B. Multiplicative models and cohort analysis. Journal of the American Statistical Association 1983; 78: 1-12. 14. Syme SL, Berkman LF. Social class: susceptibility and sickness. Am J Epidemiol 1976; 104: 1-8.

15. Antonovsky A. Social class, life expectancy and overall mortality. Milbank Mem Fund Q 1967; 45: 31-73.

16. Klag MJ, Whelton PK, Coresh J, Grim CE, Kuller LH. The association of skin color with blood pressure in US blacks with low socioeconomic status. JAMA 1991; 265: 599-602.

17. Hayward RA, Shapiro MF, Freeman HE, Corey CR. Inequities in health services among insured Americans. Do working-age adults have less access to medical care than the elderly? N Engl J Med 1988; 318: 1507-12.

18. McMahon LF, Tedeschi PJ, Wolfe RA, Griffith JR, McLaughlin CG. Small area analysis of gastrointestinal disease hospital discharge variation: are the poor at risk? J Clin Gastroenterol 1990; 12: 132-9.

19. Lurie N, Ward NB, Shapiro MF, Brook RH. Termination of Medi-Cal: does it affect health? N Engl J Med 1984; 311: 480-4.

20. Lurie N, Ward NB, Shapiro MF, et al. Termination of Medi-Cal benefits. A follow-up one year later. N Engl J Med 1985; 314: 1266-8. 